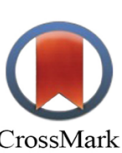

\title{
Effects of Probiotic and Prebiotic Supplementation on Leptin, Adiponectin, and Glycemic Parameters in Non-alcoholic Fatty Liver Disease: A Randomized Clinical Trial
}

\author{
Vahideh Behrouz ${ }^{1}$, Shima Jazayeri ${ }^{2, *}$, Naheed Aryaeian ${ }^{1}$, \\ Mohamad Javad Zahedi ${ }^{3}$, Fatemeh Hosseini ${ }^{4}$
}

1. Department of Nutrition, School of Public Health, Iran University of Medical Sciences, Tehran, Iran.

2. Research Center for Prevention of Cardiovascular Disease, Iran University of Medical Sciences, Tehran, Iran.

3. Digestive Research Center, Kerman University of Medical Sciences, Kerman, Iran.

4. Department of Epidemiology and Biostatistics, School of Public Health, Iran University of Medical Sciences, Tehran, Iran.

\section{* Corresponding Author:}

Shima Jazayeri M.D., Ph.D., Research Center for Prevention of Cardiovascular Disease, Iran University of Medical Sciences, Tehran, Iran. Tel: + 982186704805 Fax: +982188622707 Email:sh_jaz@yahoo.com

Received: 07 Apr. 2017 Accepted: 18 Jun. 2017

\section{ABSTRACT}

\section{BACKGROUND}

According to previous studies, probiotic and prebiotic supplementation have desirable effects on glycemic parameters. Thus far, the effect of supplementation on the glycemic parameters and adipokines in non-alcoholic fatty liver disease (NAFLD) has not been assessed. Therefore, the aim of this study was to determine the effects of supplementation with probiotic and prebiotic on adiokines and glycemic parameters in the patients with NAFLD.

\section{METHODS}

In the present randomized, double-blind, placebo-controlled trial, 89 patients with NAFLD were randomly divided into three groups to receive one probiotic capsule $+16 \mathrm{~g} / \mathrm{d}$ maltodextrin (probiotic group) or $16 \mathrm{~g} / \mathrm{d}$ oligofructose powder + one placebo capsule (prebiotic group), and one placebo capsule $+16 \mathrm{~g} / \mathrm{d}$ maltodextrin (control group) for 12 weeks. All the subjects in the study were advised to follow the weight loss diet and physical activity recommendations during the intervention. Fasting blood samples were taken at baseline and after the intervention to measure leptin, adiponectin, insulin, and fasting blood sugar.

\section{RESULTS}

At the end of the study, serum concentrations of leptin, insulin, and HOMA-IR decreased significantly in the probiotic and prebiotic groups compared with the control group. Despite the changes within the groups, serum concentrations of adiponectin did not change significantly between the three groups. Also, fasting blood sugar did not change between the groups, but decreased in the prebiotic group. Quantitative insulin-sensitivity check index (QUICKI) increased significantly in probiotic and prebiotic groups compared with the control group.

\section{CONCLUSION}

Probiotic and prebiotic supplementation along with lifestyle intervention has a favorable impact on glycemic parameters and leptin levels compared with lifestyle intervention alone.

\section{KEYWORDS:}

Probiotic, Prebiotic, Fatty liver, Insulin sensitivity, Adipokine.

Please cite this paper as:

Behrouz V, Jazayeri S, Aryaeian N, Zahedi MJ, Hosseini F. Effects of Probiotic and Prebiotic Supplementation on Leptin, Adiponectin, and Glycemic Parameters in Non-alcoholic Fatty Liver Disease: A Randomized Clinical Trial. Middle East J Dig Dis 2017;9:150-157. DOI: 10.15171/mejdd.2017.66.

\section{INTRODUCTION}

In the past few decades, non-alcoholic fatty liver disease (NAFLD) has become one of the most common causes of chronic liver disease among adults and children in developed countries. ${ }^{1}$ It includes a wide range of diseases that are associated with the excessive accumulation of fat (triglycerides) in the liver of people who do not abuse alcohol. It is also accompanied by necrosis, inflammation, and sometimes fibrosis. ${ }^{1,2}$ The global prevalence of NAFLD varies between 
$2.8 \%$ and $46 \%{ }^{3}$ In Iran, its prevalence is estimated from $21.5 \%$ to $31.5 \%$. Comorbid symptoms such as fatigue and pain negatively affect the quality of life. The high prevalence of this chronic, incurable disease imposes an economic burden on the society. ${ }^{4}$

The pathogenesis of the disease is based on insulin resistance, oxidative stress, and pro-inflammatory cytokines..$^{5-7}$ The development of hepatic steatosis is a result of increased flow of free fatty acids in the liver, reduced fatty acid beta-oxidation, and reduced secretion of triglyceriderich lipoproteins, which are the consequences of insulin resistance. ${ }^{8}$ Currently, new studies have identified other factors in the pathogenesis of NAFLD. The effect of the gut microbiota is not limited to the gastrointestinal tract and it has been recently reported that NAFLD may be associated with small intestinal bacterial overgrowth., ${ }^{9,10}$ The interaction between gut microbiota and NAFLD is supported by five mechanisms: 1) Increased ethanol production by intestinal bacteria, 2) increased metabolism of dietary choline and its deficiency (required for the synthesis of very low density lipoprotein [VLDL] and liver lipid removal), 3) release of lipopolysaccharide (LPS), 4) regulation of bile acid metabolism and FXR(Farnesoid X Receptor) signaling, and 5) increased obesity by improving the energy efficiency of food and increased fat storage. LPS, like ethanol, stimulates the production of inflammatory cytokines through a mechanism dependent on NF-k $\beta$ (Nuclear Factor- $k \beta$ ). Alteration of gut microbiota leads to increased intestinal permeability, endotoxemia, inflammation, and metabolic disorders. ${ }^{9}{ }^{91}$ According to various studies, several factors affect the intestinal immune system, including probiotics (live microorganisms) and prebiotics (food ingredients). ${ }^{12,13}$ Modification of microbial population (through the use of probiotics and prebiotics) can reduce obesity and body fat by decreasing intestinal permeability and eliminating endotoxemia. Furthermore, the growth of Bifidobacterium improves glucose sensitivity and decreases the production of pro-inflammatory mediators. ${ }^{14,15}$ Thus, the modification of microbial population can reverse metabolic disorders associated with small intestinal bacterial overgrowth. ${ }^{16}$

The objective of the present study was to assess the effects of probiotic and prebiotic supplementation on adipokines and insulin resistance in patients with NAFLD. Several studies have evaluated the effects of prebiotic supplementation on biochemical parameters associated with obesity, glucose intolerance, dyslipidemia, and inflammatory diseases. ${ }^{17,18}$ In just one study with a small sample size $(n=7)$, prebiotic supplementation effects were studied in NAFLD. ${ }^{8}$ Previous studies have reported the effects in experimental models or diseases other than fatty liver. In addition, the effect of probiotic and prebiotic consumption on adipokines and glycemic parameters has not been assessed in patients with NAFLD, lending further importance to this study's objective. Therefore, based on the efficacy of prebiotic supplementation for weight and body fat percentage ${ }^{19}$, insulin resistance, and improved dyslipidemia, we hypothesized that prebiotic supplementation would have a beneficial effect on these parameters in patients with NAFLD.

\section{MATERIALS AND METHODS}

\section{Patients}

Eligible patients referring to Be'sat Clinic, Kerman, Iran, participated in this study from January 2015 to December 2015. The study was explained to the patients and written informed consent was obtained. The study protocol was approved by the Ethics Committee of Iran University of Medical Sciences (No. 93-03-27-24996). The patients were 20 - 60 years old and NAFLD diagnosis was based on high levels of alanine aminotransferase (ALT $>1.5 \times$ upper limit of normal) and steatosis on ultrasonography (greater than grade II).$^{20}$ Other inclusion criteria were: body mass index $\geq 25 \mathrm{~kg} / \mathrm{m}^{2}$ and $\leq 40 \mathrm{~kg} / \mathrm{m}^{2}$ and no history of weight loss surgery in the past year and weight loss in the last 3 months. The exclusion criteria were pregnancy, lactation, consumption of $\omega-3$ fatty acids and nutritional supplements in the previous year, having other chronic and acute disorders of the liver (hepatitis B, C, etc.), cirrhosis, celiac disease, diabetes, hypertension, cardiovascular disease, kidney disease, lung disease, alcohol abuse, use of antibiotics over a week during the study, contraceptives pills, corticosteroids, NSAIDs, and other drugs, and also significant changes in the recommended diet and daily physical activity.

The sample size was determined according to serum insulin levels as a key variable in a previous study among the subjects with NAFLD. ${ }^{21}$ Considering the confidence level of $95 \%$ and $80 \%$ power, we needed 30 patients for each group. However, we recruited 35 patients per group to account for the possible dropouts. 


\section{Research Design}

Eligible individuals were referred from Be'sat Clinic, Kerman, Iran to participate in the study. Full information on the subject, objectives, and implementation of the study was presented in the briefing. Then, the patients signed an informed consent, completed a demographic questionnaire, and were stratified according to their age and sex. In the present study, the patients were randomly allocated into three groups according to the pre-arranged balanced block randomization to receive either one capsule probiotic plus 8 gr prebiotic placebo (maltodextrin) twice daily, or $8 \mathrm{gr}$ prebiotic powder (ORAFTI P95, BENEO, Belgium) twice daily plus one capsule probiotic placebo or 8 gr prebiotic placebo (maltodextrin) twice per day plus one capsule probiotic placebo for 12 weeks. The placebo was identical in appearance to the relevant supplements. Each probiotic capsule (Webber Naturals, Canada) contained 5 billion of 5 bacterial strains ( $\mathrm{Lac}$ tobacillus casei, Lactobacillus rhamnosus, Lactobacillus acidophilus, Bifidobacterium longum, and Bifidobacterium breve). The study was double-blind and, because the probiotic capsules and prebiotic powder were not identical, we used a double-dummy technique to blind the patients and the investigators who were assessing them. Compliance was estimated by counting pills and weighing powders. Patients were considered compliant if they consumed more than $90 \%$ of the supplements throughout the study in the three groups.

Fasting blood samples were obtained at 8:00 AM after 12-14 hours of fasting at the baseline and end of intervention. The patients completed a food-record questionnaire (two usual days and one weekend day) and international physical activity questionnaire (IPAQ) to measure the intensities of physical activity based on metabolic equivalents (MET) in weeks $0,4,8$, and 12 . In addition, at the beginning and end of the study, anthropometric measures including height (at the baseline), weight, and body fat percentage were measured. The patients' BMI was calculated via dividing weight by height squared $\left(\mathrm{kg} / \mathrm{m}^{2}\right)$. Since weight loss and increased physical activity are the main treatment for NAFLD, all the patients were offered the diet and physical activity recommendations according to the Practical Guide Identification, Evaluation, and Treatment of Overweight and Obesity in Adults from National Institute of Health. ${ }^{22}$

\section{Laboratory Methods}

Serum was isolated and frozen at $-86^{\circ} \mathrm{C}$ until analysis. Adiponectin and leptin were measured by enzyme-linked immunosorbent assay (ELISA). ELISA kits were obtained from Biovendor, Czech Republic. The intra-assay coefficient of variation for leptin and adiponectin was $4.2 \%$ and $3.9 \%$, respectively. The sensitivity for leptin and adiponectin was 0.2 and $26 \mathrm{ng} / \mathrm{mL}$, respectively. Serum insulin levels were determined by ELISA method and Demeditec kit (Germany). The intra-assay coefficient of variation for insulin was $2.6 \%$ and the sensitivity of insulin kit was $1.76 \mu \mathrm{Lu} / \mathrm{mL}$. Fasting glucose concentrations were obtained using the GOD/POD method (Pars Azmoun, Iran). All the samples were analyzed in the same batch. Insulin resistance (HOMA-IR) was calculated using the following formula ${ }^{23}$ : $[F B S(m g / d L) \times$ fasting insulin $(\mu U / m L)] / 405$. Also, quantitative insulin-sensitivity check index (QUICKI) was calculated to show insulin sensitivity by the below formula ${ }^{24}: 1 /$ [log Fasting insulin $(\mu U / m L)+\log$ Fasting glucose $(m g / d L)]$

\section{Statistical Analysis}

Collected information by food-record questionnaire was analyzed in the Nutritionist 4 software modified for Iranian foods. Kolmogorov-Smirnov test was used to verify the normal distribution. Analysis of data was performed using SPSS software version 21 for Windows (SPSS Inc, Chicago, IL, USA). To detect differences in metabolic characteristics and to determine the effect of probiotic capsule and oligofructose powder on some adipokines and glycemic parameters among the three groups, one-way analysis of variance (ANOVA) with Bonferroni post hoc was used. We adjusted all analysis for baseline values using analysis of covariance (ANCOVA). Comparison between the variables was performed by paired $t$ test at the beginning and end of the study in each group. The tests were two-sided and $\mathrm{P}$ value of less than 0.05 was considered as statistically significant.

\section{RESULTS}

89 out of the 111 patients completed 12 weeks of the study and had blood measurements at baseline and after 12 weeks. In the probiotic group, one patient was lost due to non-compliance and five patients were lost 
Table 1: Personal characteristics and body measurements in the intervention and placebo groups at the beginning

\begin{tabular}{lccc}
\hline Variables & Probiotic $(\mathbf{n}=\mathbf{3 0})$ & Prebiotic $(\mathbf{n}=\mathbf{2 9})$ & Placebo (n=30) \\
\hline Age $(\mathrm{y})$ & $38.46 \pm 7.11$ & $38.41 \pm 9.21$ & $38.43 \pm 10.09$ \\
\hline Sex $($ number $\%)$ & & $20(69 \%)$ & ns \\
\hline Male & $22(73.3 \%)$ & $9(31 \%)$ & $9(30 \%)$ \\
\hline Female & $8(26.7 \%)$ & $89.16 \pm 12.69$ & $90.65 \pm 14.78$ \\
\hline Weight $(\mathrm{kg})$ & $84.98 \pm 11.75$ & $30.81 \pm 4.74$ & $31.90 \pm 5.04$ \\
\hline BMI $\left(\mathrm{kg} / \mathrm{m}^{2}\right)$ & $29.56 \pm 2.54$ & $31.38 \pm 6.64$ & $32.18 \pm 6.41$ \\
\hline Body fat percentage & $30.23 \pm 5.33$ & $\mathrm{~ns}$ \\
\hline
\end{tabular}

Values are reported as mean $\pm \mathrm{SD}$.

* Obtained from the ANOVA test for comparison of data among the intervention and placebo groups.

Ns: not significant.

Table 2: Anthropometric measurements and physical activity at baseline and after 12 weeks

\begin{tabular}{|c|c|c|c|c|c|}
\hline Parameter & Studied group & Week 0 & Week 12 & $P$ value* & $P$ value $* *$ \\
\hline \multirow[t]{3}{*}{ Weight (Kg) } & Probiotic & $84.98 \pm 11.7$ & $80.32 \pm 10.8$ & $<0.001$ & \multirow{3}{*}{0.104} \\
\hline & Prebiotic & $89.16 \pm 12.6$ & $84.76 \pm 11.9$ & $<0.001$ & \\
\hline & Placebo & $90.65 \pm 14.7$ & $87.36 \pm 15.1$ & $<0.001$ & \\
\hline \multirow[t]{3}{*}{$\mathrm{BFP}$} & Probiotic & $30.23 \pm 5.3$ & $28.1 \pm 5.2$ & $<0.001$ & \multirow{3}{*}{0.190} \\
\hline & Prebiotic & $31.38 \pm 6.6$ & $29.25 \pm 6.9$ & $<0.001$ & \\
\hline & Placebo & $32.18 \pm 6.4$ & $30.94 \pm 5.9$ & 0.007 & \\
\hline \multirow[t]{3}{*}{$\mathrm{BMI}\left(\mathrm{Kg} / \mathrm{m}^{2}\right)$} & Probiotic & $29.56 \pm 2.5$ & $27.98 \pm 2.4$ & $<0.001$ & \multirow{3}{*}{0.081} \\
\hline & Prebiotic & $30.81 \pm 4.7$ & $29.31 \pm 4.7$ & $<0.001$ & \\
\hline & Placebo & $31.9 \pm 5.04$ & $30.66 \pm 4.7$ & $<0.001$ & \\
\hline \multirow[t]{3}{*}{ Energy (Kcal) } & Probiotic & $2388.8 \pm 542.4$ & $1854.1 \pm 379.7$ & $<0.001$ & \multirow{3}{*}{0.798} \\
\hline & Prebiotic & $2527.9 \pm 681.7$ & $1917.2 \pm 384.6$ & $<0.001$ & \\
\hline & Placebo & $2417.1 \pm 706.5$ & $1909.9 \pm 422.1$ & $<0.001$ & \\
\hline \multirow[t]{3}{*}{$\operatorname{MET}(\mathrm{m} / \mathrm{d})$} & Probiotic & $231.3 \pm 265.3$ & $727 \pm 446.4$ & $<0.001$ & \multirow{3}{*}{0.604} \\
\hline & Prebiotic & $272.5 \pm 320.1$ & $641.6 \pm 297.6$ & $<0.001$ & \\
\hline & Placebo & $283.2 \pm 418.2$ & $769.3 \pm 664.7$ & 0.001 & \\
\hline
\end{tabular}

Values are reported as mean $\pm \mathrm{SD}$.

* Obtained from the paired samples $t$ test for comparison of data between the beginning and end of the study.

**Obtained from ANCOVA test adjusted for the baseline values among the intervention and placebo groups.

BFP: body fat percentage, BMI: body mass index, MET: metabolic equivalent

to follow-up. In the prebiotic group, six patients were lost to follow-up, one withdrew because of rhinoplasty surgery, three were excluded because of travel, and two refused to give blood after the intervention. In the placebo group, one patient was excluded due to pregnancy and three were lost to follow-up. Therefore, the results obtained with 30 patients in the probiotic group, 29 patients in prebiotic group, and 30 patients in placebo groups. No serious adverse reactions were reported after consumption of the probiotic and prebiotic supplementation in the patients with NAFLD throughout the study. The compliance for probiotic, prebiotic, and placebo groups was $96.5 \%, 95.5 \%$, and $96 \%$ respectively. As shown in table 1 , there were no significant differences in body mass index, weight, body fat percentage, age, and sex among the groups at baseline (table 1).

Weight, body fat percentage, body mass index, physical activity, and energy intake are shown in table 2 . There was a significant difference between the value of these variables at the beginning and end of the intervention within each group, but these changes were not significant between the three studied groups (table 2).

According to the data shown in table 3, leptin, insulin, and HOMA-IR concentrations decreased significantly in 
Table 3: Adipokines and glycemic parameters before and after intervention

\begin{tabular}{|c|c|c|c|c|c|c|c|c|}
\hline Parameter & & $\begin{array}{l}\text { Probiotic } \\
(n=30)\end{array}$ & $P^{*}$ & $\begin{array}{c}\text { Prebiotic } \\
(n=29)\end{array}$ & $P^{*}$ & $\begin{array}{c}\text { Placebo } \\
(n=30)\end{array}$ & $P^{*}$ & $P^{* *}$ \\
\hline \multirow{3}{*}{$\begin{array}{l}\text { Leptin } \\
(\mathrm{ng} / \mathrm{mL})\end{array}$} & Before & $73.10 \pm 26.78$ & \multirow{3}{*}{$<0.001$} & $80.34 \pm 29.71$ & \multirow{3}{*}{$<0.001$} & $75.85 \pm 26.96$ & \multirow{3}{*}{0.629} & \multirow{3}{*}{$0.000 \dagger$} \\
\hline & & & & & & & & \\
\hline & After & $48.6 \pm 13.56$ & & $56.86 \pm 22.83$ & & $74.41 \pm 26.17$ & & \\
\hline \multirow{3}{*}{$\begin{array}{l}\text { Adiponectin } \\
(\mu \mathrm{g} / \mathrm{mL})\end{array}$} & Before & $24.36 \pm 11.11$ & \multirow{3}{*}{$<0.001$} & $27.82 \pm 10.43$ & \multirow{3}{*}{$<0.001$} & $25.78 \pm 9.45$ & \multirow{3}{*}{0.005} & \multirow{3}{*}{0.837} \\
\hline & & & & & & & & \\
\hline & After & $40.73 \pm 24.14$ & & $43.9 \pm 15.66$ & & $39.37 \pm 24.17$ & & \\
\hline \multirow{3}{*}{ FBS } & Before & $91.47 \pm 9.86$ & \multirow{3}{*}{0.349} & $95.31 \pm 17.67$ & \multirow{3}{*}{0.026} & $98.3 \pm 24.16$ & \multirow{3}{*}{0.368} & \multirow{3}{*}{0.296} \\
\hline & & & & & & & & \\
\hline & After & $88.83 \pm 10.14$ & & $89.41 \pm 16.76$ & & $96.23 \pm 20.62$ & & \\
\hline \multirow{3}{*}{$\begin{array}{c}\text { Insulin } \\
(\mu \mathrm{IU} / \mathrm{mL})\end{array}$} & Before & $16.7 \pm 8.72$ & \multirow{3}{*}{0.004} & $15.06 \pm 6.26$ & \multirow{3}{*}{$<0.001$} & $17.44 \pm 11.66$ & \multirow{3}{*}{0.382} & \multirow{3}{*}{$0.004 \dagger$} \\
\hline & & & & & & & & \\
\hline & After & $12.92 \pm 9.32$ & & $11.42 \pm 4.50$ & & $18.49 \pm 12.76$ & & \\
\hline \multirow{3}{*}{ HOMA-IR } & Before & $3.82 \pm 2.14$ & \multirow{3}{*}{0.01} & $3.62 \pm 1.89$ & \multirow{3}{*}{$<0.001$} & $4.7 \pm 5.87$ & \multirow{3}{*}{0.959} & \multirow{3}{*}{$0.026 \dagger$} \\
\hline & & & & & & & & \\
\hline & After & $2.96 \pm 2.53$ & & $2.58 \pm 1.35$ & & $4.8 \pm 5.45$ & & \\
\hline \multirow{3}{*}{ QUICKI } & Before & $0.32 \pm 0.024$ & \multirow{3}{*}{0.001} & $0.32 \pm 0.021$ & \multirow{3}{*}{$<0.001$} & $0.31 \pm 0.024$ & \multirow{3}{*}{0.614} & \multirow{3}{*}{$0.000 \dagger$} \\
\hline & & & & & & & & \\
\hline & After & $0.33 \pm 0.031$ & & $0.34 \pm 0.023$ & & $0.31 \pm 0.022$ & & \\
\hline
\end{tabular}

Values are reported as mean \pm SD.

P*: Obtained from the paired samples t test for comparison of data between the beginning and end of the study.

$\mathrm{P}^{* *}$ : Obtained from ANCOVA test adjusted for the baseline values among the intervention and placebo groups.

$\dagger$ : Significant difference between probiotic and prebiotic groups with placebo group that obtained from Bonferoni post hoc

the probiotic and prebiotic groups at the end of week 12 compared with the baseline (leptin: $p<0.001$ in both intervention groups, insulin: $p<0.01$ in probiotic group, and $p<0.001$ in prebiotic group, and HOMA-IR: $p<0.05$ in probiotic group and $p<0.01$ in prebiotic group) and also among the groups after the treatment $(p<0.001, p<0.01$, and $p<0.05$, respectively). Serum concentrations of FBS and adiponectin did not change significantly based on ANCOVA ( $p=0.296$ and $p=0.837$, respectively). But, adiponectin increased significantly in all groups $(p<0.01)$. Fasting blood glucose levels showed no significant difference in the placebo and probiotic groups at the end of the study, but there was a significant reduction in the prebiotic group $(p<0.05)$. QUICKI increased significantly in prebiotic and probiotic groups at the end of the study and among the three groups $(p=0.001)$.

\section{DISCUSSION}

Based on our review of the literature, this study was the first randomized, double-blind clinical trial that investigated the effect of prebiotic and probiotic supple- mentation on the level of adipokines including leptin and adiponectin, and glycemic parameters in the patients with NAFLD in conjunction with lifestyle modification (diet and exercise recommendations). The current study demonstrated that administration of probiotic and prebiotic among the patients with NAFLD for 12 weeks had beneficial effects on insulin metabolism and serum leptin; however, it did not affect adiponectin and fasting blood sugar compared with the placebo group. Few studies have investigated the effects of probiotics and prebiotics on adipokines such as leptin and adiponectin. In agreement with our study, An and colleagues ${ }^{25}$ showed the effect of Bifidobacterium (B. pseudocatenulatum SPM 1204, $B$. longum SPM 1205, and B. longum SPM 1207) to reduce circulating leptin in obese mice. Administration of Lactobacillus plantarum also reduced serum levels of leptin in Takemura and co-workers' animal study. ${ }^{26}$ In another work, Parnell and others ${ }^{27}$ found oligofructose supplementation reduced weight, body fat percentage, fasting glucose, insulin, and leptin levels due to the reduction of adipose tissue in adults with overweight/ 
obesity. In contrast to our study, in Hume's study ${ }^{28}$ after 16 weeks of supplementation with prebiotic fiber among overweight and obese children, there was a significant increase in adiponectin concentration, while leptin levels did not change between the studied groups.

In one study, Kadooka and colleagues ${ }^{29}$ used probiotic supplement (L. gasseri SBT 2055) to improve obesity and abdominal obesity in adults. L. gasseri SBT 2055 supplementation significantly reduced abdominal visceral fat and increased serum adiponectin in obese people. In addition, Kishino and co-workers ${ }^{30}$ showed that Salacia reticulata supplementation increased the plasma levels of adiponectin in type 2 diabetic rats. In another study, Lactobacillus plantarum strain No. 14 reduced white adipose tissue without changing the adiponectin concentration in high fat fed mice. ${ }^{26}$

The exact mechanisms by which prebiotic and probiotic might influence adipokines concentrations are unknown, but studies suggest that their influence is mediated by changes in the microbiota. ${ }^{14}$ Lactobacillus stimulates the production of certain cytokines such as TNF- $\alpha$ and, thus, can be effective in regulating the gene expression of leptin. ${ }^{26,31}$ Furthermore, the production of SCFAs (Short Chain Fatty Acids) during the fermentation of prebiotic fiber may have a positive effect on adiponectin secretion. ${ }^{32}$ In our study, although in the probiotic and prebiotic groups, the amount of adiponectin increase was larger than that of placebo group, it was not statistically significant possibly because of the effect of lifestyle intervention in each group.

Insulin resistance is a feature of NAFLD and metabolic syndrome. To the best knowledge of the authors, there are no studies that have investigated the effects of probiotic and prebiotic supplementation in one trial. In the current work, HOMA-IR, QUICKI, and serum insulin concentrations were significantly changed in the probiotic and prebiotic groups compared with the baseline levels in the study and placebo groups. Most of the previous studies have shown that treatment with prebiotic or probiotic can control glycemia or decrease serum insulin ${ }^{33-37}$, however, some findings are inconsistent. ${ }^{38-40}$ In agreement with our study, Daubioul and colleagues ${ }^{8}$ observed a significant reduction in the insulin concentration following the intake of oligofructose among the patients with NAFLD after 4 weeks. In another study ${ }^{41}$, the effect of probiotic supplementation on improving glycemic parameters dem- onstrated a significant decrease in fasting glucose, insulin, and HOMA-IR levels compared with the placebo group. In contrast, Dehghan and co-workers ${ }^{42}$ showed oligofructose-enriched inulin supplementation reduced fasting blood glucose in the intervention group compared with the placebo. Also, fasting insulin and HOMA-IR were not reduced in the intervention group compared with the placebo. The difference in the results of human studies ${ }^{39,42-44}$ could be related to the differences in the kind of studied diseases, quantity and composition of the basal microbiota population, type of supplements and dosage, basic levels of glycemic parameters, duration of the intervention, and sample size. In the current study, non-significant changes of FBS may be attributed to small sample size.

Possible mechanisms by which prebiotic intake might improve insulin resistance include the adjustment of the energy metabolism, control of obesity, and increased production of GLP-1 and GLP-2 (Glucagon-like peptide 1 and Glucagon-like peptide 2 respectively). GLP-1 moderates pancreatic and plasma insulin secretion, beta cell mass, and its function. Also, GLP-2 enhances insulin sensitivity in the liver, fat, and muscle.$^{38}$ Probiotics are effective in improving the insulin resistance by reducing the concentration of endotoxin, increasing fecal $\mathrm{PH}$, and reducing the production and absorption of intestinal toxins. ${ }^{45}$ Thus, modulating gut microbiota can be effective in improving glycemic status through the use of probiotics and prebiotics.

The strengths of this study were the blocked randomization stratified of patients and assessment of adipokines secreted from adipose tissue to assess the effects of probiotic and prebiotic supplementation on the adipose tissue. Also, the participants' disease was re-assessed recently and none of them had received any treatment. The other strength of this study was the comparison of prebiotic and probiotic supplementations effect on the investigated variables. The limitations of this study were the impracticality of assessing liver fibrosis and inflammatory biomarkers at the cellular level due to limited financial resources. Fecal bacteria loads were not quantified before and after the treatment. Also, because of time and budget limitations, there was no possibility of increasing the duration of the intervention, sample size, or investigation of simultaneous prebiotic and probiotic supplementation (synbiotic) in a distinct group. The appli- 
cation of this study is that probiotic and prebiotic supplementation can be recommended as a therapeutic aid for lifestyle modification in the subjects with NAFLD.

\section{CONCLUSION}

This double-blind randomized controlled clinical trial shows that probiotic and prebiotic supplementation along with lifestyle intervention creates favorable changes in glycemic parameters and leptin levels compared with the lifestyle intervention alone. Also in this study, oligofructose dietary fiber intake was as effective as probiotic supplementation in controlling insulinemia and adipokines, but further studies are needed to confirm this issue.

\section{ACKNOWLEDGEMENTS}

The present study was supported by a grant from Vice Chancellor for Research, Iran University of Medical Sciences, Tehran, Iran (no. 24996). Thanks are extended to Digestive Research Center (KMU, Kerman, Iran) for their assistance in this project.

Clinical trial registration number: www.irct. ir:IRCT201410052394N13.

\section{CONFLICT OF INTEREST}

The authors declare no conflict of interest related to this work.

\section{REFERENCES}

1. Della Corte C, Liccardo D, Mosca A, Vania A, Nobili V. Non-alcoholic fatty liver disease. Paediatr Child Health 2013;23:529-34. doi: 10.1016/j.paed.2013.08.003.

2. Ostad Rahimi A, Mahdavi R, Somi MH, Tarzemani MK. Oxidative stress-related parameters and antioxidant status in nonalcoholic fatty liver disease patients. IJEM 2011;12:493-9.

3. Corrado RL, Torres DM, Harrison SA. Review of treatment options for nonalcoholic fatty liver disease. Med Clin North Am 2014;98:55-72. doi: 10.1016/j.mena.2013.09.001.

4. Ghaemi A, Taleban F, Hekmatdoost A, Rafiei A, Hosseini V, Amiri Z, et al. Effect of weight reduction diet on non-alcoholic fatty liver disease. Iran J Nutr Sci Food Technology 2013;8:123-34.

5. Lam B, Younossi ZM. Review: treatment options for nonalcoholic fatty liver disease. Therap Adv Gastroenterol 2010;3:121-37. doi: 10.1177/1756283X09359964.

6. Guo H, Zhong R, Liu Y, Jiang X, Tang X, Li Z, et al. Effects of bayberry juice on inflammatory and apoptotic markers in young adults with features of non-alcoholic fatty liver disease. Nutrition 2014;30:198-203. doi:10.1016/j.nut.2013.07.023.

7. Eslamparast T, Eghtesad S, Hekmatdoost A, Poustchi H. Probiotics and nonalcoholic fatty liver disease. Middle
East J Dig Dis 2013;5:129-36.

8. Daubioul C, Horsmans Y, Lambert P, Danse E, Delzenne NM. Effects of oligofructose on glucose and lipid metabolism in patients with nonalcoholic steatohepatitis: results of a pilot study. Eur J Clin Nutr 2005;59:723-6. doi:10.1038/sj.ejen.1602127.

9. Compare D, Coccoli P, Rocco A, Nardone O, De Maria S, Cartenì M, et al. Gut-liver axis: the impact of gut microbiota on non alcoholic fatty liver disease. Nutr Metab Cardiovasc Dis 2012;22:471-6. doi:10.1016/j.numecd.2012.02.007.

10. Aron-Wisnewsky J, Gaborit B, Dutour A, Clement K. Gut microbiota and non-alcoholic fatty liver disease: new insights. Clin Microbiol Infect 2013;19:338-48. doi: http:10.1111/1469-0691.12140.

11. Nicolucci AC, Hume MP, Reimer RA. Effect of Prebiotic Fiber Intake on Adiposity and Inflammation in Overweight and Obese Children: Assessing the Role of the Gut Microbiota. Can J Diabetes 2015;39:S43. doi: 10.1016/j.jcjd.2015.01.166

12. Vieira AT, Teixeira MM, Martins FS. The role of probiotics and prebiotics in inducing gut immunity. Front Immunol 2013;4:445. doi:10.3389/fimmu.2013.00445.

13. Jakobsdottir G, Nyman M, Fåk F. Designing future prebiotic fiber to target metabolic syndrome. Nutrition 2014;30:497-502. doi:10.1016/j.nut.2013.08.013.

14. Savcheniuk OA, Virchenko OV, Falalyeyeva TM, Beregova TV, Babenko LP, Lazarenko LM, et al. The efficacy of probiotics for monosodium glutamate-induced obesity: dietology concerns and opportunities for prevention. EPMA J 2014;5:2. doi: 10.1186/1878-5085-5-2.

15. Bernini LJ, Simão ANC, Alfieri DF, Lozovoy MAB, Mari NL, de Souza CHB, et al. Beneficial effects of Bifidobacterium lactis on lipid profile and cytokines in patients with metabolic syndrome: A randomized trial. Effects of probiotics on metabolic syndrome. Nutrition 2016;32:716-9. doi:10.1016/j.nut.2015.11.001.

16. Geurts L, Neyrinck AM, Delzenne NM, Knauf C, Cani PD. Gut microbiota controls adipose tissue expansion, gut barrier and glucose metabolism: novel insights into molecular targets and interventions using prebiotics. Benef Microbes 2014;5:3-17. doi: 10.3920/BM2012.0065.

17. Parnell JA, Raman M, Rioux KP, Reimer RA. The potential role of prebiotic fibre for treatment and management of non-alcoholic fatty liver disease and associated obesity and insulin resistance. Liver Int 2012;32:701-11. doi:10.1111/j.1478-3231.2011.02730.x.

18. Kellow NJ, Coughlan MT, Reid CM. Metabolic benefits of dietary prebiotics in human subjects: a systematic review of randomised controlled trials. $\mathrm{Br} J \mathrm{Nutr}$ 2014;111:1147-61. doi: 10.1017/S0007114513003607.

19. Liber A, Szajewska H. Effects of inulin-type fructans on appetite, energy intake, and body weight in children and adults: systematic review of randomized controlled trials. Ann Nutr Metab 2013;63:42-54. doi:10.1159/000350312.

20. Mazhar SM, Shiehmorteza M, Sirlin CB. Noninvasive assessment of hepatic steatosis. Clin Gastroenterol Hepatol 2009;7:135-40. doi:10.1016/j.cgh.2008.11.023.

21. Bugianesi E, Pagotto U, Manini R, Vanni E, Gastaldelli A, de Iasio R, et al. Plasma adiponectin in nonalcoholic fatty 
liver is related to hepatic insulin resistance and hepatic fat content, not to liver disease severity. J Clin Endocrinol Metab 2005;90:3498-504. doi:10.1210/jc.2004-2240.

22. National Heart L, and Blood Institute. The practical guide: identification, evaluation, and treatment of overweight and obesity in adults. National institutes of Heart, National Heart, Lung, and Blood Institute, NHLBI Obesity Education Initiative, North American Association for the Study of Obesity, 2000.

23. Matthews D, Hosker J, Rudenski A, Naylor B, Treacher D, Turner R. Homeostasis model assessment: insulin resistance and $\beta$-cell function from fasting plasma glucose and insulin concentrations in man. Diabetologia 1985;28:412-9. doi:10.1007/BF00280883.

24. Katz A, Nambi SS, Mather K, Baron AD, Follmann DA, Sullivan G, et al. Quantitative insulin sensitivity check index: a simple, accurate method for assessing insulin sensitivity in humans. $J$ Clin Endocrinol Metab 2000;85:2402-10. doi: 10.1210/jcem.85.7.6661.

25. An HM, Park SY, Lee DK, Kim JR, Cha MK, Lee SW, et al. Antiobesity and lipid-lowering effects of Bifidobacterium spp. in high fat diet-induced obese rats. Lipids Health Dis 2011;10:116. doi: 10.1186/1476-511X-10-116.

26. Takemura N, Okubo T, Sonoyama K. Lactobacillus plantarum strain No. 14 reduces adipocyte size in mice fed high-fat diet. Exp Biol Med 2010;235:849-56. doi: 10.1258/ebm.2010.009377.

27. Parnell JA, Reimer RA. Weight loss during oligofructose supplementation is associated with decreased ghrelin and increased peptide YY in overweight and obese adults. Am J Clin Nutr 2009;89:1751-9. doi: 10.3945/ajcn.2009.27465.

28. Hume M. The Effects of Prebiotic Fibre Intake on Appetite and Body Mass Index z-score in Overweight and Obese Children. (Doctoral dissertation, University of Calgary, 2015).

29. Kadooka Y, Sato M, Imaizumi K, Ogawa A, Ikuyama K, Akai $Y$, et al. Regulation of abdominal adiposity by probiotics (Lactobacillus gasseri SBT2055) in adults with obese tendencies in a randomized controlled trial. Eur $J$ Clin Nutr 2010;64:636-43. doi:10.1038/ejen.2010.19.

30. Kishino E, Ito T, Fujita K, Kiuchi Y. A mixture of Salacia reticulata (Kotala himbutu) aqueous extract and cyclodextrin reduces body weight gain, visceral fat accumulation, and total cholesterol and insulin increases in male Wistar fatty rats. Nutr Res 2009;29:55-63. doi:10.1016/j.nutres.2008.11.001.

31. Sato M, Uzu K, Yoshida T, Hamad EM, Kawakami H, Matsuyama H, et al. Effects of milk fermented by Lactobacillus gasseri SBT2055 on adipocyte size in rats. $\mathrm{Br} J$ Nutr 2008;99:1013-7. doi:10.1017/S0007114507839006.

32. Xiong Y, Miyamoto N, Shibata K, Valasek MA, Motoike T, Kedzierski RM, et al. Short-chain fatty acids stimulate leptin production in adipocytes through the $\mathrm{G}$ protein-coupled receptor GPR41. Proc Natl Acad Sci U $S$ A 2004;101:1045-50. doi: 10.1073/pnas.2637002100.

33. Genta S, Cabrera W, Habib N, Pons J, Carillo IM, Grau A, et al. Yacon syrup: beneficial effects on obesity and insulin resistance in humans. Clin Nutr 2009;28:182-7. doi:10.1016/j.clnu.2009.01.013.

34. Russo F, Riezzo G, Chiloiro M, De Michele G, Chimienti $\mathrm{G}$, Marconi E, et al. Metabolic effects of a diet with inulin- enriched pasta in healthy young volunteers. Curr pharm des 2010;16:825-31. doi: 10.2174/138161210790883570.

35. Vulevic J, Juric A, Tzortzis G, Gibson GR. A mixture of trans-galactooligosaccharides reduces markers of metabolic syndrome and modulates the fecal microbiota and immune function of overweight adults. $J$ Nutr 2013;143:324-31. doi: 10.3945/jn.112.166132.

36. Karimi P, Farhangi MA, Sarmadi B, Gargari BP, Zare Javid A, Pouraghaei M, et al. The therapeutic potential of resistant starch in modulation of insulin resistance, endotoxemia, oxidative stress and antioxidant biomarkers in women with type 2 diabetes: a randomized controlled clinical trial. Ann Nutr Metab 2016;68:85-93. doi: 10.1159/000441683.

37. Abbaszadeh S, Parastouei K, Afshari H, Rostami L, Mahmoudi H, Gamarchehreh M, et al. Effects of multistrain probiotic supplementation on glycemic and inflammatory indices in patients with nonalcoholic fatty liver disease: a double-blind randomized clinical trial. J Am Coll Nutr 2016;35:500-5. doi: 10.1080/07315724.2015.1031355.

38. Pourghassem Gargari B, Dehghan P, Aliasgharzadeh A, Asghari Jafar-abadi M. Effects of high performance inulin supplementation on glycemic control and antioxidant status in women with type 2 diabetes. Diabetes Metab J 2013;37:140-8. doi:10.4093/dmj.2013.37.2.140.

39. Dewulf EM, Cani PD, Claus SP, Fuentes S, Puylaert PG, Neyrinck AM, et al. Insight into the prebiotic concept: lessons from an exploratory, double blind intervention study with inulin-type fructans in obese women. Gut 2013;62:1112-21. doi: 10.1136/gutjnl-2012-303304.

40. Yousefinejad A, Mazloom Z, Dabbaghmanesh MH. Effect of probiotics on lipid profile, glycemic control, insulin action, oxidative stress, and inflammatory markers in patients with type 2 diabetes: a clinical trial. Iran JMed Sci 2013;38:38-43.

41. Rajkumar H, Mahmood N, Kumar M, Varikuti SR, Challa HR, Myakala SP. Effect of probiotic (VSL\# 3) and omega-3 on lipid profile, insulin sensitivity, inflammatory markers, and gut colonization in overweight adults: a randomized, controlled trial. Mediators Inflamm 2014;2014:348959. doi:10.1155/2014/348959.

42. Dehghan P, Gargari BP, Jafar-Abadi MA, Aliasgharzadeh A. Inulin controls inflammation and metabolic endotoxemia in women with type 2 diabetes mellitus: a randomized-controlled clinical trial. Int J Food Sci Nutr 2014;65:117-23. doi: 10.3109/09637486.2013.836738.

43. Luo J, Van Yperselle M, Rizkalla SW, Rossi F, Bornet FR, Slama G. Chronic consumption of short-chain fructooligosaccharides does not affect basal hepatic glucose production or insulin resistance in type 2 diabetics. J Nutr 2000;130:1572-7.

44. De Luis D, De la Fuente B, Izaola O, Conde R, Gutiérrez S, Morillo M, et al. Double blind randomized clinical trial controlled by placebo with an alpha linoleic acid and prebiotic enriched cookie on risk cardiovascular factor in obese patients. Nutr Hosp 2011;26:827-33. doi:10.1590/ S0212-16112011000400024.

45. Eslamparast T, Poustchi H, Zamani F, Sharafkhah M, Malekzadeh R, Hekmatdoost A. Synbiotic supplementation in nonalcoholic fatty liver disease: a randomized, double-blind, placebo-controlled pilot study. Am J Clin Nutr 2014;99:535-42. doi: 10.3945/ajen.113.068890. 